\title{
Intra-patient dose escalation in Ewing's sarcoma treated with vincristine, doxorubicin, cyclophosphamide alternating with ifosfamide and etoposide: a retrospective review
}

\author{
Jeremy Lewin ${ }^{1 *}$, Samantha Wieringa², Marnie Collins ${ }^{1}$, Jayesh Desai', Lisa Orme', Senthil Lingaratnam²
} and David M Thomas ${ }^{1}$

\begin{abstract}
Background: Data suggests that males experience less toxicity and poorer survival than females treated for Ewing's sarcoma. We instituted an intra-patient dose escalation (DE) policy with Vincristine/Doxorubicin/Cyclophosphamide (VDC) alternating with Ifosfamide/Etoposide (IE) based on hematological nadirs and report its feasibility and safety.

Methods: A retrospective review of adherence to DE guidelines and toxicities was conducted for patients who received DE with VDC/IE over 3 years at a single cancer center. Absolute neutrophil counts (ANC) was collected on days 8,12 and 15 for cycles $1-6$. DE of $10 \% /$ cycle was applied if ANC $>1.5 \times 10^{9} / \mathrm{L}$ and platelet $>100 \times 10^{9} / \mathrm{L}$ on all blood results. The primary endpoint was the proportion of patients who received appropriate DE. The secondary endpoint was to assess morbidity, changes in hematologic nadirs between gender and age and a comparison with a prior cohort of ESFT patients who did not receive DE. Gender comparisons were assessed via independent 2-sample t-tests assuming unequal variances. Within cycle changes in hematologic nadirs were assessed using repeated measures ANOVA. Relapse free survival and overall survival (OS) curves were estimated using the Kaplan-Meier method.

Results: 23 patients were identified (mean age: 27; range 17-54). 91 decisions for DE were made (1 decision excluded because of progressive disease) with $90 \%$ concordance with guidelines. No adverse outcomes occurred as a result of the inappropriate escalation. Grade 3/4 febrile neutropenia (FN) during VDC and IE was 26.1\% (6/23 patients) and $17.4 \%$ respectively with no difference for those who were DE. Males were less neutropenic after C1 and $C 3$ of VDC compared to females ( $P$-value $C 1=0.003 ; C 3=0.005$ ). VDC was associated with greater neutropenia on day 8 whereas IE had greater neutropenia on day 12 (P-value $<0.001$ ). During VDC, a non statistical difference in neutropenia was seen for individuals aged 15-25 $(n=13)$ compared with older individuals (P-value $=0.09)$. OS comparison for those with localized disease with a prior cohort who were not DE showed similar outcomes (P-value $=0.37$ )
\end{abstract}

Conclusions: DE is deliverable without increased adverse outcomes. Males have less myelosuppression during VDC, and should be especially considered for DE.

Keywords: Ewing's sarcoma, Dose escalation, Toxicity, Neutropenia, Chemotherapy

\footnotetext{
* Correspondence: Jeremy.Lewin@petermac.org

'Sarcoma Service, Peter MacCallum Cancer Centre, Locked Bag 1, A'Beckett

Street, Melbourne, VIC 8006, Australia

Full list of author information is available at the end of the article
} 


\section{Background}

Ewing sarcoma family tumors (ESFT) are malignant tumors of bone and soft tissue. Patients presenting with localized disease are usually treated with multimodality approach, given high relapse rates (80-90\%) without chemotherapy $[1,2]$. Modern treatment plans include neo-adjuvant and adjuvant multi-agent chemotherapy, to target the high risk of subclinical metastatic disease at the time of diagnosis. In studies predominantly involving pediatric populations, this approach leads to 5 year survival rates around 70\% [3-5]. Even in patients presenting with metastatic disease, longterm survival may be seen in $20 \%$ of patients [2]. The traditional chemotherapy used in both localized and metastatic disease is Vincristine, Doxorubicin, Cyclophosphamide alternating with Ifosfamide and Etoposide (VDC/IE) [2,6], with suggestions that the dose of doxorubicin may be related to outcome [7].

Despite a steady improvement in cancer care generally, there has been a discouraging lack of improvement in survival rates in adolescent and young adults (AYA) [8]. For patients diagnosed with ESFT between the ages 1530 , survival is approximately half that of children with males having increased mortality compared to females in the AYA subgroup [9-11]. There has been much speculation as to the reasons for these observations. The suggestion that relative underdosing of AYA compared to children may play a part is supported by data showing that adults with pediatric-type sarcomas experience less treatment-related toxicity compared with their younger counterparts [10,12-14]. A meta-analysis in osteosarcoma showed that children had higher rates of neutropenia compared with adolescent or adult patients, and that dose-related toxicities including thrombocytopenia and mucositis were strongly associated with improved overall survival [9]. In addition to age, gender-related differences also exist in osteosarcoma, with males experiencing less myelosuppression and inferior survival $[7,9]$. A retrospective study of 14,824 patients showed that male AYA patients with osteosarcoma, Ewing sarcoma and Hodgkin lymphoma experienced less toxicity, lower response rates and excess mortality relative to children and female counterparts [12]. Similar findings correlating lack of chemotherapy-induced toxicity and inferior outcome [15-17], have also been reported in testis, breast and ovarian cancers [18].

These data suggest that age and gender-related differences in drug handling may account for some of the survival and toxicity differences in the AYA population. If so, dose intensification may improve outcomes in chemosensitive cancers. Accurate dosing of chemotherapy is difficult using traditional algorithms based on body surface area [19], with evidence for systematic underdosing [20,21]. While one study of dose escalation of alkylating agents did not improve outcomes in Ewing sarcoma [22], increasing dose density from a 21 day cycle to a 14 day cycle with growth factor support [23] may be associated with improved survival without increased toxicity [24]. These approaches have relied on dose intensification across all patients treated on these protocols, rather than intrapatient dose modification to achieve a pharmacodynamic effect. Current studies of anthracycline pharmacokinetics and pharmacodynamics (ACTRN12609000956202) will aid in justifying and developing strategies for "individualizing" chemotherapy, enabling the use of readily available surrogate markers such as the degree of myelosuppression, or measures of the antiproliferative activity of drugs, to guide dose-adjustments $[25,26]$.

In the absence of more accurate algorithms for chemotherapy dosing, one practical solution for personalized, intra-patient dosing is "toxicity-adjusted" dosing [20]. In 2009, we implemented a policy at the Peter MacCallum Cancer Centre to individualized dose escalation of chemotherapy in Ewing sarcoma patients, using nadir bloods counts as the primary measure of a dose-related pharmacodynamic effect. We now report on our dose escalation (DE) policy over a 3 year period to assess overall deliverability, safety and treatment related toxicity.

\section{Methods}

The primary objective was to assess the proportion of patients that received $\mathrm{DE}$ and the proportion of $\mathrm{DE}$ decisions that were appropriate according to the protocol. The secondary objective was to assess rates of serious complications for the entire group, investigate gender and age related differences in neutrophil and platelet nadirs, assess subsequent neutrophil and platelet nadirs for those who underwent dose escalation, and calculate relapse free survival (RFS) and overall survival (OS). In order to assess RFS and OS, a comparison was performed with a prior cohort of ESFT with localized disease treated with the same regimen of VDC/IE, but prior to the initiation of our dose escalation policy. This study was approved by the Institutional Review Board of Peter MacCallum Cancer Centre for retrospective data collection.

Chemotherapy was delivered for ESFT every 3 weeks with vincristine $(2 \mathrm{mg})$, doxorubicin $\left(75 \mathrm{mg} / \mathrm{m}^{2}\right)$, cyclophosphamide $\left(1200 \mathrm{mg} / \mathrm{m}^{2}\right)$ D1 alternating with ifosfamide $\left(1800 \mathrm{mg} / \mathrm{m}^{2}\right)$ and etoposide $\left(100 \mathrm{mg} / \mathrm{m}^{2}\right)$ D1-5 [2]. Blood counts were taken on Day 8, 12 and 15 for the first 6 cycles of chemotherapy and dose escalation (DE) was implemented if the neutrophil and platelet count did not fall below $1.5 \times 10^{9} / \mathrm{L}$ or $100 \times 10^{9} / \mathrm{L}$, respectively, in the absence of non-hematologic toxicities of concern. Although these hematological cut-offs have not been validated in prospective trials, these values have been suggested in the literature in recommendations for "toxicity adjusted dosing" $[15,18]$. Dose escalation involved increasing the BSA calculated dose of all chemotherapy agents, except vincristine, by $10 \%$ for all equivalent subsequent cycles. Given 
the alternating regimen, nadir counts for cycle 1 and 2 led to DE decision for cycle 3 and 4, whereas nadir counts for cycle 3 and 4 led to a DE decision in cycle 5 and 6 . Deescalation occurred on the basis of both hematological and non-hematological toxicities at the discretion of the treating physician.

\section{Inclusion}

All patients with ESFT (including peripheral primitive neuroectodermal tumor, Askin's tumors, Ewing's sarcoma, desmoplastic small round cell tumor) who underwent cytotoxic chemotherapy at Peter MacCallum with VDC/IE between March 2009 and August 2012 were included.

\section{Statistical analysis}

Analysis of primary endpoints and secondary endpoints was performed on an intention to treat basis. To address the primary objective, the proportion of patients who received DE was calculated for each of cycles $3-6$ separately as well as for any VDC cycle (3 and/or 5) and any IE cycle ( 4 and/or 6$)$. To evaluate adherence, the proportion of treatment decisions that were appropriate according to the DE protocol was calculated.

To address the secondary objectives, the proportion of patients reporting grade $3 / 4$ febrile neutropenia in each of the VDC and IE cycles was calculated. Comparisons between males and females in terms of neutrophil and platelet nadirs in cycles $1-4$ were investigated using independent 2-sample t-tests assuming unequal variances. Within cycle changes in neutrophil and platelet counts as well as between cycle changes in neutrophil and platelet nadirs were assessed using repeated measures ANOVA. Overall survival was measured as the time from first chemotherapy until death and relapse-free survival was measured from time of first chemotherapy until date of relapse. Patients were censored at the last date they were known to be alive and relapse-free respectively. OS and RFS curves were estimated using the Kaplan-Meier product-limit method. OS and RFS comparison to the prior historical cohort was assessed using the logrank test. A $P$-value of $<0.05$ was considered statistically significant. Statistical analysis was performed using Excel, GraphPad Prism 6 and R version 2.15.2 software.

\section{Results}

Between 2009 and 2012, 23 patients were treated on the DE protocol with a median follow up of $2.3(0.7-3.4)$ years. Baseline demographics are shown in Table 1. In total, 91 events for consideration of dose escalation occurred ( 1 decisions was excluded because of progressive disease). This is presented in Table 2. There were 3 patients during VDC that were escalated twice. Although not statistically significant, more males had DE than
Table 1 Baseline demographics

\begin{tabular}{lc}
\hline Baseline demographics & \\
\hline Number & $10(43 \%)$ \\
Female & $13(57 \%)$ \\
Male & $23(17-54)$ \\
Median age (range) & $21(17-54)$ \\
Median age female & $26(18-40)$ \\
Median age male & \\
Diagnosis & $17(74 \%)$ \\
EWINGS & $5(22 \%)$ \\
PNET & $1(4 \%)$ \\
DSRCT & \\
Disease site & $6(26 \%)$ \\
Metastatic & \\
Localized & $9(39 \%)$ \\
Extremity & $2(9 \%)$ \\
Pelvic & $2(9 \%)$ \\
Axial trunk & $3(13 \%)$ \\
Chest wall & $1(4 \%)$ \\
Other & $1.79(\mathrm{SD} \pm 0.22)$ \\
BSA & $1.94(\mathrm{SD} \pm 0.15)$ \\
Male &
\end{tabular}

females during VDC (7/13 vs $3 / 10)$, but not during IE (7/13 vs $5 / 10)$.

Protocol adherence was recorded at $90 \%$. Nine events of non-adherence to the protocol were recorded (7 events of inappropriate escalation (7.7\%) and 2 events of missed escalation (2.2\%); details of inappropriate escalation are shown in Additional file 1: Table S1). No serious adverse outcomes occurred as a result of the inappropriate escalation. The rate of Grade 3/4 febrile neutropenia (FN) during VDC was $26.1 \%$ (6/23 patients) with median length of

Table 2 Number of patients who received dose escalation during VDC/IE

\begin{tabular}{|c|c|c|c|c|}
\hline & Escalated & Dose unchanged & Dose reduced & Number \\
\hline \multicolumn{5}{|l|}{ VDC } \\
\hline $\mathrm{C} 1$ & & & & 23 \\
\hline $\mathrm{C} 3$ & 10 & 10 & 3 & 23 \\
\hline $\mathrm{C} 5$ & 3 & 19 & 1 & 23 \\
\hline Any cycle & 10 & 10 & 3 & 23 \\
\hline \multicolumn{5}{|l|}{ IE } \\
\hline $\mathrm{C} 2$ & & & & 23 \\
\hline $\mathrm{C} 4$ & 10 & 11 & 2 & 23 \\
\hline $\mathrm{C} 6$ & 3 & 19 & 0 & 22 \\
\hline Any cycle & 12 & 9 & 2 & 23 \\
\hline
\end{tabular}


stay (LOS) of 4 days (range 1-20) with no ICU admissions. The rate of Grade 3/4 FN for IE was $17.4 \%$ (4/23 patients) with a median LOS of 4 days (3-7) and 1 ICU admission. The ICU stay occurred in a patient who was appropriately not dose-escalated. There were no deaths related to chemotherapy and only 1 patient received a platelet transfusion (during IE). There were no additional documented grade $3 / 4$ toxicities documented in those who underwent dose escalation.

Neutrophil nadirs by gender for cycle $1-4$ are shown in Table 3. Males were statistically significantly less neutropenic after $\mathrm{C} 1$ and $\mathrm{C} 3$ of VDC compared to females $(P$-value $\mathrm{C} 1=0.003 ; \mathrm{C} 3=0.005)$. VDC was associated with greater neutropenia on day 8 (ANC: D8: $0.98 \pm 0.97 \times$ $10^{9} / \mathrm{L}, P$-value $\left.<0.001\right)$ whereas IE had greater neutropenia on day 12 (ANC: D12: $3.74 \pm 3.04 \times 10^{9} / \mathrm{L}, P$-value $<0.001$ ). Although not statistically significant, there was also a trend for less thrombocytopenia during VDC in males (data not shown, $P$-value $=0.11$ ). After cycle 1 of VDC, individuals aged $15-25(n=13)$ had lower neutrophil nadirs compared with older individuals (mean difference $=0.62 \times$ $10^{9} / \mathrm{L}, P$-value $=0.15$ ) although this was not statistically significant. Differences in cytopenia in IE dosing according to gender were only seen in cycle 4 (mean difference = $2.31 \times 10^{9} / \mathrm{L}, P$-value $\left.=0.05\right)$ (Table 3$)$.

Although the numbers were too small to achieve statistical significance, dose escalation, whether appropriate or not, was associated with a reduction in neutrophil count in subsequent cycles. For those who received dose escalation in cycle 1 of VDC, the mean nadir ANC decreased from $1.59 \pm 1.22 \times 10^{9} / \mathrm{L}$ to $1.29 \pm 1.07 \times 10^{9} / \mathrm{L}$ in cycle 3 , and $1.04 \pm 0.9 \times 10^{9} / \mathrm{L}$ in cycle $5(P$-value $=0.29)$. For IE cycles, those who received dose escalation after cycle 2 showed a decrease from $4.82 \pm 1.88 \times 10^{9} / \mathrm{L}$ to $2.10 \pm 1.8 \times 10^{9} / \mathrm{L}$ in cycle 4 . Using current dosing algorithms, dose escalation resulted in a trend to males receiving a higher relative dose intensity of VDC and IE compared to females (VDC: $107 \%$ vs $98 \%, P$-value $=0.12$; IE: $105 \%$ vs $102 \%, P$-value $=0.49$ ).

To compare overall cancer-related outcomes between the traditional BSA associated chemotherapy dosing and the toxicity-adapted dose escalation protocol, we compared the RFS and OS between treated patients with localized disease before (1995-2004) and during (20092013) the introduction of the DE policy. The baseline characteristics of this historical comparator group were similar to our cohort (An additional file shows this in more detail [see Additional file 2: Table S2]) and patients with DSRCT and metastatic disease were excluded from this analysis. The 2 year RFS in the dose escalation cohort was $88 \%$, compared to $63 \%$ in the historical comparator cohort $(\mathrm{HR}=0.33$, 95\% CI: $(0.07-1.56) ; P$-value $=$ 0.14 ) and 2 year overall survival was $88 \%$ vs $75 \%$ in the historical comparator cohort $(\mathrm{HR}=0.48,95 \% \mathrm{CI}$ : $(0.09$ 2.46) $P$-value $=0.37$ (Figures 1 and 2). When making comparisons in our cohort group only, there was no statistically significant difference in RFS $(P$-value $=0.29)$ and OS $(P$-value $=0.31)$ when separated by those who have had DE (for either VDC or IE) compared to those who did not in patients with localized disease.

\section{Discussion}

The key finding in this study is that individualized dose escalation of chemotherapy in ESFT is feasible in a single sarcoma unit. Less neutropenia was observed in males, and perhaps in older patients, during the anthracycline cycles, suggesting that BSA-based dosing alone may not be adequate in males. The protocol compliance rate was 90\%. Given the experimental nature of a toxicity-adjusted protocol, we continually reviewed the safety and compliance after implementation of the protocol. Importantly, no short or long term adverse outcomes were seen in this

Table 3 Gender related difference in nadir blood counts after C1 and C3

\begin{tabular}{|c|c|c|c|c|c|c|c|c|c|}
\hline & \multicolumn{6}{|c|}{ Gender } & \multirow{3}{*}{$\begin{array}{c}\text { Mean } \\
\text { difference }\end{array}$} & \multirow[t]{3}{*}{$95 \% \mathrm{Cl}$} & \multirow[t]{3}{*}{$P$-value } \\
\hline & \multicolumn{3}{|c|}{ Male } & \multicolumn{3}{|c|}{ Female } & & & \\
\hline & $N$ & Mean & SD & $N$ & Mean & SD & & & \\
\hline \multicolumn{10}{|c|}{ Neutrophil nadir } \\
\hline \multicolumn{10}{|l|}{ VDC } \\
\hline Cycle 1 & 13 & 1.56 & 1.35 & 9 & 0.16 & 0.19 & 1.40 & $(0.57,2.21)$ & 0.003 \\
\hline Cycle 3 & 12 & 1.59 & 1.08 & 9 & 0.42 & 0.56 & 1.17 & $(0.40,1.93)$ & 0.005 \\
\hline \multicolumn{10}{|l|}{ IE } \\
\hline Cycle 2 & 13 & 3.77 & 2.70 & 10 & 4.40 & 4.59 & -0.63 & $(-4.14,2.88)$ & 0.71 \\
\hline Cycle 4 & 12 & 3.17 & 3.54 & 9 & 0.86 & 1.18 & 2.31 & $(-0.04,4.66)$ & 0.05 \\
\hline \multicolumn{10}{|c|}{ Dose intensity (\%) } \\
\hline VDC & 13 & 107 & 9 & 10 & 98 & 16 & 9 & $(-3,22)$ & 0.12 \\
\hline IE & 13 & 105 & 7 & 10 & 102 & 11 & 3 & $(-5,11)$ & 0.49 \\
\hline
\end{tabular}

$P$-values correspond to comparisons between genders. Dose intensity of VDC and IE is compared to baseline. 


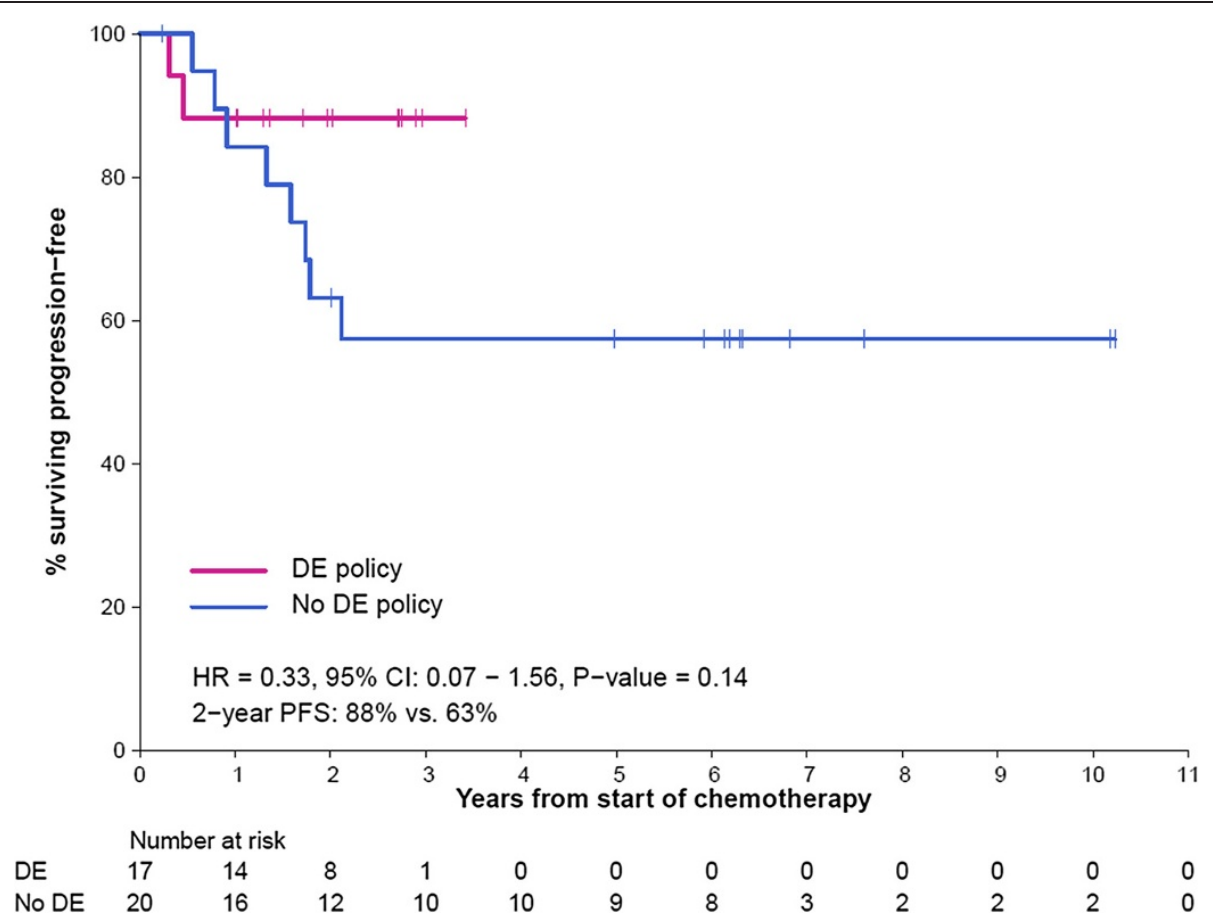

Figure 1 Relapsed-free survival for localized ESFT compared between those who underwent a DE policy (2009 - 2012) compared with a prior cohort who did not have a DE policy (1995 - 2004) showing a HR=0.33 (95\% Cl: $(0.07-1.53) P$-value 0.14$)$.

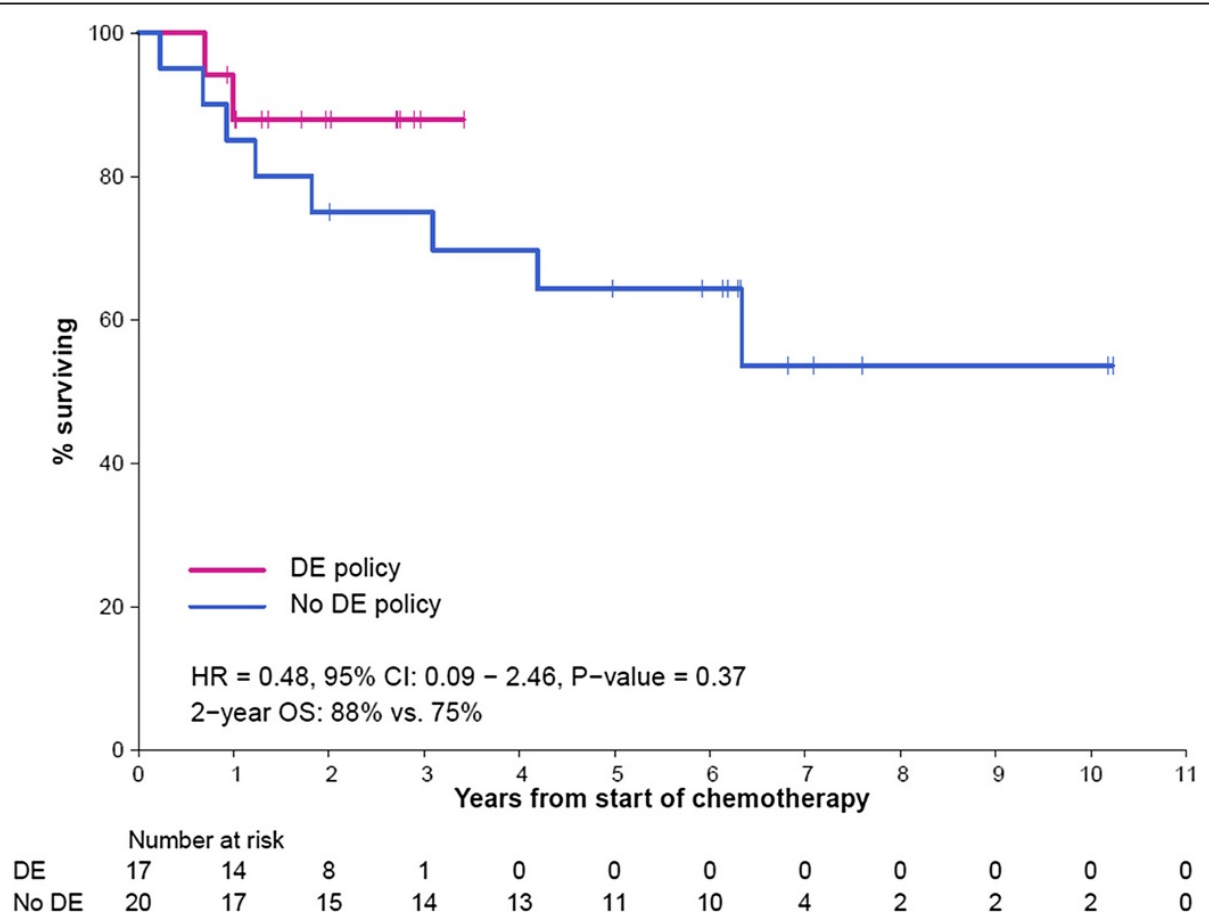

Figure 2 Overall survival for localized ESFT compared between those who underwent a DE policy (2009 - 2012) compared with a prior cohort who did not have a DE policy $(1995-2004)$ showing a $\mathrm{HR}=0.48(95 \% \mathrm{Cl}$ : $(0.09-2.46) P$-value 0.37$)$. 
study as a result of intrapatient dose escalation. The RFS and OS comparison to the prior ESFT cohort suggests that dose escalation is not associated with worse overall outcomes for patients with localized primary ESFT. Clearly, safe dose escalation requires a formal program with strictly defined criteria for escalation, close monitoring of nadir blood counts, and patient selection limited to those without other dose-related toxicities or co-morbid illnesses. Given this study showed gender and age related differences in cytopenia, it is arguable that dose escalation should be especially considered in older males during the anthracycline component of treatment (Cycle 3 or 5 based on C1 nadir). Additionally, variation was seen in the timing of neutropenia nadirs, which may have ramifications for the timing of pegylated filgrastim delivery.

It is important to put these findings into context given evidence for gender- and age-related difference in toxicity and outcome in chemo-sensitive cancers such as ESFT [12]. Although the peak incidence of ESFT is during the AYA years, these patients have a worse survival compared to a pediatric population [27]. The reasons behind this comprise a combination of biological differences [27,28], delayed diagnosis, poorer treatment compliance [29,30], psychosocial overlay and limited involvement in clinical trials [31,32]. With regards to biological differences, in the EURO-Ewing 99 protocol [10], increasing age and male gender was associated with less toxicity using VIDE chemotherapy, a protocol similar to VDC/IE. Additionally, gender related differences have previously been described in hodgkins lymphoma [33], osteosarcoma [9,34,35] and ewings sarcoma $[12,36,37]$. A meta-analysis in osteosarcoma confirmed differences in outcome related to gender and age and showed improved outcomes in females, and those with Grade 3/4 mucositis [9]. Interestingly, it also confirmed the results of our study showing increased myelosuppression in the younger age group although given our small numbers, this was not statistically significant. Collectively, this data suggests a better understanding of cytotoxic pharmacology is needed in the AYA population $[28,38]$, and may be used to improve cancer outcomes.

Adolescent and young adult patients undergo genderspecific pubertal changes in body composition, size and hormonal status, which may alter the pharmacokinetic/ pharmacodynamic properties of chemotherapy [38]. Young women have significant increase in fat mass during adolescent years, with subsequent higher body fat than men [39]. Higher fat distribution has effects on drug clearance and volume of distribution $[28,40]$ which may cause variations in the dose of chemotherapy delivered. That being said, a large pediatric study in ALL showed no differences in toxicity, survival or PK data in the obese patient [41]. It is difficult to isolate drug-specific effects in complex, multi-drug chemotherapeutic regimens, and it is important to recognize that different drugs may demonstrate different pharmacologic profiles in the AYA population. Nearly all the benefit of methotrexate's use in osteosarcoma has been demonstrated in patients under the age of 40 [42] and doxorubicin, a key component of regimens used to treat ESFT, osteosarcoma and hodgkins disease, demonstrates significant pharmacokinetic variation according to gender and body habitus $[43,44]$. It is possible that the inferior outcomes of EFST in older males may be attributed to relative doxorubicin under-dosing of these individuals.

Given the inherent inaccuracies in current dosing algorithms, and the importance of chemotherapy to cure for chemo-sensitive cancers, intra-patient dose modification based on markers of toxicity provides a practical strategy for individually tailored dosing $[25,26]$. In this context, this limited study suggests gender- and potentially age-related differences in hematological nadirs, and that intra-patient dose escalation appears safe and feasible. It is important to note that this retrospective study only comprises 23 patients, with relatively short follow up. In particular, the non-significant trend to improved RFS and OS in the dose escalation group will require larger numbers of patients and longer follow up. Additionally, we did not assess long-term morbidity, including the potential for cumulative anthracycline cardiotoxicity in a young population.

\section{Conclusion}

Individualized, toxicity-adapted dosing of chemotherapy, added to an initial calculation of dosing based on BSAalone, aims to correct the gender and age related disparity in outcomes, without relying on new chemotherapeutic agents. Pharmacological data in this age group is lacking [28] and adequate dose-response is critical for chemosensitive diseases such as ESFT, germ cell tumors and Hodgkin's in maximizing outcome where a dose-response relationship exists. The key finding in this study is that individualized dose escalation of chemotherapy in ESFT is feasible in a single sarcoma unit without increased adverse outcomes. Additionally we have showed that less neutropenia was observed in males, and perhaps in older patients, during the anthracycline cycles and should be especially considered for DE. Given the rarity of ESFT presents significant statistical challenges in stratifying gender and age differences, pharmacological endpoints should be designed into future prospective chemotherapy trials.

\section{Additional files}

Additional file 1: Table S1. Description of patients who inappropriately received dose escalation.

Additional file 2: Table S2. Comparison of baseline demographics with for patients with localized disease who underwent a DE policy (2009 - 2012) compared with a prior cohort who did not have a DE policy $(1995$ - 2004). Patients in both cohorts were treated with alternating VDC/IE. 


\section{Abbreviations}

ANC: Absolute neutrophil count; AYA: Adolescent and young adult; DE: Dose escalation; ESFT: Ewing's sarcoma family tumors; FN: Febrile neutropenia; ICU: Intensive care unit; IE: Ifosfamide, etoposide; VDC: Vincristine, doxorubicin, cyclophosphamide; LOS: Length of stay; OS: Overall survival; RFS: Relapse free survival.

\section{Competing interests}

The authors declare that they have no competing interests.

\section{Authors' contributions}

$J \mathrm{~L}$ and DT conceived and designed the study. SW and $J \mathrm{~L}$ undertook the data retrieval. JL, DT and MC analyzed the data. All authors participated in writing of the manuscript. All authors read and approved the final manuscript.

\section{Author details}

'Sarcoma Service, Peter MacCallum Cancer Centre, Locked Bag 1, A'Beckett Street, Melbourne, VIC 8006, Australia. ${ }^{2}$ Pharmacy Department, Peter MacCallum Cancer Centre, Melbourne, Australia.

Received: 6 November 2013 Accepted: 3 December 2013

Published: 10 December 2013

\section{References}

1. Nesbit ME: Ewing's sarcoma. CA Cancer J Clin 1976, 26:174-180.

2. Grier HE, Krailo MD, Tarbell NJ, Link MP, Fryer CJ, Pritchard DJ, Gebhardt MC, Dickman PS, Perlman EJ, Meyers PA, et al: Addition of ifosfamide and etoposide to standard chemotherapy for Ewing's sarcoma and primitive neuroectodermal tumor of bone. N Engl J Med 2003, 348:694-701.

3. Craft A, Cotterill S, Malcolm A, Spooner D, Grimer R, Souhami R, Imeson J, Lewis I: Ifosfamide-containing chemotherapy in Ewing's sarcoma: the second united kingdom children's cancer study group and the medical research council Ewing's tumor study. J Clin Oncol 1998, 16:3628-3633.

4. Paulussen M, Ahrens S, Dunst J, Winkelmann W, Exner GU, Kotz R, Amann G, Dockhorn-Dworniczak B, Harms D, Muller-Weihrich S, et al: Localized ewing tumor of bone: final results of the cooperative Ewing's sarcoma study CESS 86. J Clin Oncol 2001, 19:1818-1829.

5. Balamuth NJ, Womer RB: Ewing's sarcoma. Lancet Oncol 2010, 11:184-192.

6. Kolb EA, Kushner BH, Gorlick R, Laverdiere C, Healey JH, LaQuaglia MP, Huvos AG, Qin J, Vu HT, Wexler L, et al: Long-term event-free survival after intensive chemotherapy for Ewing's family of tumors in children and young adults. J Clin Oncol 2003, 21:3423-3430.

7. Burgert EO Jr, Nesbit ME, Garnsey LA, Gehan EA, Herrmann J, Vietti TJ, Cangir A, Tefft M, Evans R, Thomas P, et al: Multimodal therapy for the management of nonpelvic, localized Ewing's sarcoma of bone: intergroup study IESS-II. J Clin Oncol 1990, 8:1514-1524.

8. Bleyer A, Choi M, Fuller CD, Thomas CR Jr, Wang SJ: Relative lack of conditional survival improvement in young adults with cancer. Semin Oncol 2009, 36:460-467.

9. Collins M, Wilhelm M, Conyers R, Herschtal A, Whelan J, Bielack S, Kager L, Kuhne T, Sydes M, Gelderblom $H$, et al: Benefits and adverse events in younger versus older patients receiving neoadjuvant chemotherapy for osteosarcoma: findings from a meta-analysis. J Clin Oncol 2013, 31:2303-2312

10. Juergens C, Weston C, Lewis I, Whelan J, Paulussen M, Oberlin O, Michon J, Zoubek A, Juergens $H$, Craft A: Safety assessment of intensive induction with vincristine, ifosfamide, doxorubicin, and etoposide (VIDE) in the treatment of Ewing tumors in the EURO-E.W.I.N.G. 99 clinical trial. Pediatr Blood Cancer 2006, 47:22-29.

11. Bleyer A, O'Leary M, Barr R, Ries L: Cancer epidemiology in older adolescents and young adults 15 to 29 years of age, including SEER incidence and survival: 1975-2000. Bethesda, MD: National Cancer Institute; 2006. No. 06-5767.

12. Khamly KK, Thursfield VJ, Fay M, Desai J, Toner GC, Choong PF, Ngan SY, Powell GJ, Thomas DM: Gender-specific activity of chemotherapy correlates with outcomes in chemosensitive cancers of young adulthood. Int J Cancer 2009, 125:426-431.

13. Gupta AA, Anderson JR, Pappo AS, Spunt SL, Dasgupta R, Indelicato DJ, Hawkins DS: Patterns of chemotherapy-induced toxicities in younger children and adolescents with rhabdomyosarcoma: a report from the children's oncology group soft tissue sarcoma committee. Cancer 2012, 118:1130-1137.
14. Ferrari S, Palmerini E, Staals E, Abate ME, Longhi A, Cesari M, Balladelli A, Pratelli L, Bacci G: Sex- and age-related chemotherapy toxicity in patients with non-metastatic osteosarcoma. J Chemother 2009, 21:205-210.

15. Gao B, Klumpen HJ, Gurney H: Dose calculation of anticancer drugs. Expert Opin Drug Metab Toxicol 2008, 4:1307-1319.

16. Canal P, Chatelut E, Guichard S: Practical treatment guide for dose individualisation in cancer chemotherapy. Drugs 1998, 56:1019-1038.

17. Cortes EP, Holland JF, Wang JJ, Sinks LF, Blom J, Senn H, Bank A, Glidewell $\mathrm{O}$ : Amputation and adriamycin in primary osteosarcoma. N Engl J Med 1974, 291:998-1000.

18. Gurney H: I don't underdose my patients... do I? Lancet Oncol 2005, 6:637-638.

19. Gurney H: Developing a new framework for dose calculation. J Clin Oncol 2006, 24:1489-1490

20. Gurney $\mathrm{H}$ : Dose calculation of anticancer drugs: a review of the current practice and introduction of an alternative. J Clin Oncol 1996, 14:2590-2611.

21. Baker SD, Verweij J, Rowinsky EK, Donehower RC, Schellens JH, Grochow LB, Sparreboom A: Role of body surface area in dosing of investigational anticancer agents in adults, 1991-2001. J Natl Cancer Inst 2002, 94:1883-1888.

22. Granowetter L, Womer R, Devidas M, Krailo M, Wang C, Bernstein M, Marina N, Leavey P, Gebhardt M, Healey J, et al: Dose-intensified compared with standard chemotherapy for nonmetastatic ewing sarcoma family of tumors: a children's oncology group study. J Clin Oncol 2009, 27:2536-2541.

23. Womer RB, Daller RT, Fenton JG, Miser JS: Granulocyte colony stimulating factor permits dose intensification by interval compression in the treatment of Ewing's sarcomas and soft tissue sarcomas in children. Eur J Cancer 2000, 36:87-94.

24. Womer R, West D, Krailo M, Dickman P, Pawel B: Randomized comparison of every-two-week v. Every-three-week chemotherapy in ewing sarcoma family tumors (ESFT). J Clin Oncol 2008, 26(supp):554s.

25. Newell DR: Getting the right dose in cancer chemotherapy-time to stop using surface area? Br J Cancer 2002, 86:1207-1208.

26. Gurney H: How to calculate the dose of chemotherapy. Br J Cancer 2002 86:1297-1302

27. Bleyer A, Barr R, Hayes-Lattin B, Thomas D, Ellis C, Anderson B: The distinctive biology of cancer in adolescents and young adults. Nat Rev Cancer 2008, 8:288-298.

28. Veal GJ, Hartford CM, Stewart CF: Clinical pharmacology in the adolescent oncology patient. J Clin Oncol 2010, 28:4790-4799.

29. Smith SD, Rosen D, Trueworthy RC, Lowman JT: A reliable method for evaluating drug compliance in children with cancer. Cancer 1979, 43:169-173

30. Mitchell AE, Scarcella DL, Rigutto GL, Thursfield VJ, Giles GG, Sexton M Ashley DM: Cancer in adolescents and young adults: treatment and outcome in Victoria. Med J Aust 2004, 180:59-62.

31. Bleyer A, Budd T, Montello M: Adolescents and young adults with cancer: the scope of the problem and criticality of clinical trials. Cancer 2006, 107:1645-1655.

32. Burke ME, Albritton K, Marina N: Challenges in the recruitment of adolescents and young adults to cancer clinical trials. Cancer 2007, 110:2385-2393.

33. Klimm B, Reineke T, Haverkamp H, Behringer K, Eich HT, Josting A, Pfistner B, Diehl $V$, Engert A: Role of hematotoxicity and sex in patients with Hodgkin's lymphoma: an analysis from the german hodgkin study group. J Clin Oncol 2005, 23:8003-8011.

34. Smeland S, Muller C, Alvegard TA, Wiklund T, Wiebe T, Bjork O, Stenwig AE, Willen $\mathrm{H}$, Holmstrom $\mathrm{T}$, Folleras $\mathrm{G}$, et al: Scandinavian sarcoma group osteosarcoma study SSG VIII: prognostic factors for outcome and the role of replacement salvage chemotherapy for poor histological responders. Eur J Cancer 2003, 39:488-494.

35. Bielack SS, Kempf-Bielack B, Delling G, Exner GU, Flege S, Helmke K, Kotz R, Salzer-Kuntschik M, Werner M, Winkelmann W, et al: Prognostic factors in high-grade osteosarcoma of the extremities or trunk: an analysis of 1,702 patients treated on neoadjuvant cooperative osteosarcoma study group protocols. J Clin Oncol 2002, 20:776-790.

36. Bacci G, Longhi A, Ferrari S, Mercuri M, Versari M, Bertoni F: Prognostic factors in non-metastatic Ewing's sarcoma tumor of bone: an analysis of 579 patients treated at a single institution with adjuvant or neoadjuvant chemotherapy between 1972 and 1998. Acta Oncol 2006, 45:469-475.

37. Gehan EA, Nesbit ME Jr, Burgert EO Jr, Viettit J, Tefft M, Perez CA, Kissane J, Hempel C: Prognostic factors in children with Ewing's sarcoma. Nat/ Cancer Inst Monogr 1981, 56:273-278. 
38. Thomas DM, Albritton $\mathrm{KH}$, Ferrari A: Adolescent and young adult oncology: an emerging field. J Clin Oncol 2010, 28:4781-4782.

39. Loomba-Albrecht LA, Styne DM: Effect of puberty on body composition. Curr Opin Endocrinol Diabetes Obes 2009, 16:10-15.

40. Cheymol G: Effects of obesity on pharmacokinetics implications for drug therapy. Clin Pharmacokinet 2000, 39:215-231.

41. Hijiya N, Panetta JC, Zhou Y, Kyzer EP, Howard SC, Jeha S, Razzouk Bl, Ribeiro RC, Rubnitz JE, Hudson MM, et al: Body mass index does not influence pharmacokinetics or outcome of treatment in children with acute lymphoblastic leukemia. Blood 2006, 108:3997-4002.

42. Anninga JK, Gelderblom H, Fiocco M, Kroep JR, Taminiau AH, Hogendoorn PC, Egeler RM: Chemotherapeutic adjuvant treatment for osteosarcoma: where do we stand? Eur J Cancer 2011, 47:2431-2445.

43. Dobbs NA, Twelves CJ, Gillies H, James CA, Harper PG, Rubens RD: Gender affects doxorubicin pharmacokinetics in patients with normal liver biochemistry. Cancer Chemother Pharmacol 1995, 36:473-476.

44. Rodvold KA, Rushing DA, Tewksbury DA: Doxorubicin clearance in the obese. J Clin Oncol 1988, 6:1321-1327.

doi:10.1186/2045-3329-3-15

Cite this article as: Lewin et al:: Intra-patient dose escalation in Ewing's sarcoma treated with vincristine, doxorubicin, cyclophosphamide alternating with ifosfamide and etoposide: a retrospective review. Clinical Sarcoma Research 2013 3:15.

\section{Submit your next manuscript to BioMed Central and take full advantage of:}

- Convenient online submission

- Thorough peer review

- No space constraints or color figure charges

- Immediate publication on acceptance

- Inclusion in PubMed, CAS, Scopus and Google Scholar

- Research which is freely available for redistribution 